\title{
Design and Development for Forgery Currency Detection using SIFT Features based SVM Classifier
}

\author{
M.Praneesh, R.Nagarajan, P.Kavitha
}

\begin{abstract}
There are many methods for identifying a fake currency notes which we have discussed and each one has its own significance. But, there is no software to detect fake currencies. The first process is to get the original and fake currency image from the data set.After getting the two images are pre-analysis the both original and fake image Convert the image into gray color. To extract the black strips in both currency images. After conversion the image segmentation are applied and the post-processing are applied. After that the feature extraction are classified undergoes SVM Classifier. SIFT Algorithm are used in the training set to count the black strips from both original and forgery image. Finally, there are two types of result will be executed under the histogram feature analysis and probability map and next one is to counting the black strips from both original and fake images.
\end{abstract}

Index Terms: SVM Classifier, fake currency, SIFT Features, Pre processing.

\section{INTRODUCTION}

Image Processing is a technique to enhance raw images received from cameras/sensors placed on satellites, space probes and aircrafts or pictures taken in normal day-today life for various applications. Various techniques have been developed in Image Processing during The last four to five decades. Most of techniques are developed for enhancing images obtained from unmanned spacecrafts, space probes and military reconnaissance flights[1]. Image Processing systems are becoming popular due to easy availability of powerful personnel computers, large size memory devices, graphics software etc Feature extraction is an individual form of dimensionality reduction in which the contribution of data's to the algorithm, where it is great to be processed and it is supposed to be disreputably redundant (e.g. the same dimension in both feet and meters) then the input data will be distorted into a compact depiction set of features (also named features vector). Transforming the input data into the set of features is called feature extraction. If the features extractions are cautiously chosen it is predictable that the features set will extract the applicable information from the input data in order to execute the preferred task using this reduced representation instead of the full size input [5]. Image segmentation is the method of partition a digital image into several segments. The objective of segmentation is to simplify and/or modify the representation of an image into impressive that is more significant and easier to analyze. Image segmentation is classically used to position objects and limitations (lines, curves, etc.) in images. More precisely, image segmentation is the development of transmission a label to every pixel in an image such that pixels with the same label split certain visual characteristics. The effect of image segmentation is a set of segments that jointly cover the complete image, or a set of contours extracted from the image. Each of the pixels in a region is comparable with reverence to some feature or computed property, such as color, intensity, or texture. Adjacent regions are significantly dissimilar with regard to the same characteristic. The goal of image segmentation is to cluster pixels into salient image regions, regions corresponding to individual surfaces, objects, or natural parts of objects.

\section{MATERIALS AND METHODS}

There are many methods for identifying a fake currency notes which we have discussed and each one has its own significance. In this paper are mainly used to survey counterfeit currency by using two main algorithms one SIFT Algorithm and next is SVMClassifier.The system will work on two images, one is Original Image of the paper currency and the other is test image on which verification is to be performed. The proposed algorithm for the discussed paper currency verification system is presented as follows.

1. Image of the paper currency will be acquired by simple scanner in .jpg extension.

2. The image processing will be implemented on this image.

3. The various characteristics of the paper currency will be cropped and segmented.

\section{A. System Flow Diagram}

The overall architecture of the proposed system is described in the figure 1 Ramakrishna college of Arts and Science, Bharathiar University.

P.Kavitha, Assistant Professor, Dept of Computer Science, Sri Ramakrishna college of Arts and Science, Bharathiar 


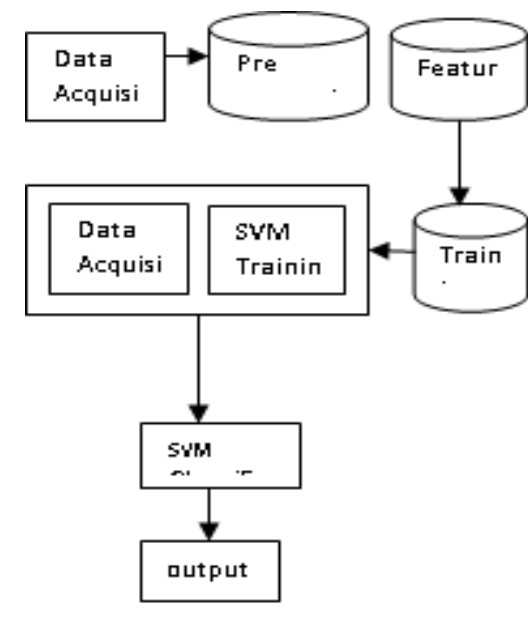

Fig-1 System Architecture

\section{B. Pre processing}

The aim of image processing is to suppress undesired distortions or enhance some image features that are important for further processing or analysis . In this process first step is to get the original and fake image currency images from the data set and pre-processing those images to adjust the image into gray scale level .It also including image acquisition, image smoothing. Edge detection is by far the common approach for detecting discontinuities the currency edges intensity values.

\section{Feature Extraction}

In this feature extraction currency to get the original currency the original and duplicate currency note features like Water Marking, Security Thread, and See through Register etc., during the feature extraction process the dimensionality of data is reduced. Extracting too many features will not only increase the cost but also sometimes lower the system performance in terms of execution time based on Texture Feature and Color Feature. In this process the currency black strips are counted from both original and fake currency by using Sift Algorithm the next step is to analysis the histogram analysis process to get the result. SIFT (Scale Invariant Features Techniques )

\section{D.SVM Classifier}

After the sift Algorithm the next step is SVM classifier Both training and test functions depend on data and kernel function. Even the need to evaluate dot product would result in less complexity of computing kernel. Thus, SVM circumvents both forms of curse of dimensionality; proliferation of parameters causing intractable complexity and over fitting. Training algorithms may take advantage of parallel processing in several ways such as, evaluation of kernel and sum are highly parallelizable procedures. SVM usually exhibit good generalization performance. SVM is an estimation algorithm i.e. learning machine based on (Burges , 1998) which has following three steps:

\section{RESULTS AND DISCUSSION}

Below fig2 shows the results of proposed approach.

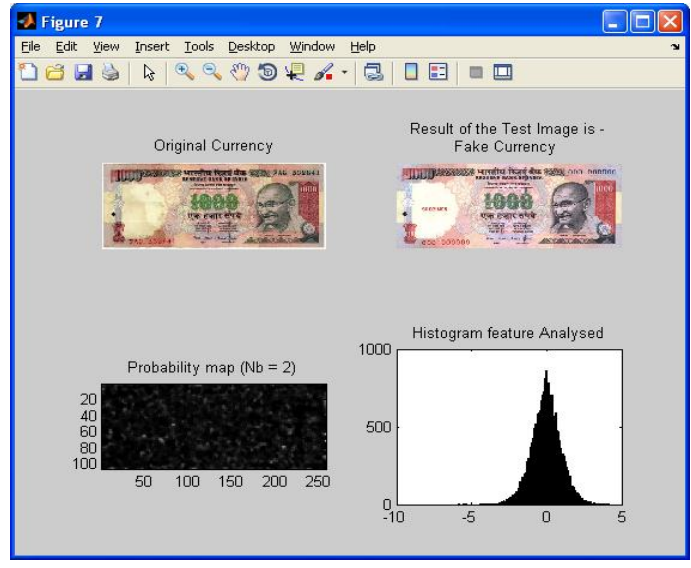

Fig 2: Results of proposed approach

In this process the original image and test image individually perform the SIFT features and find the probability map for the required images. If it is same then classify the image based on SVM Classifier. For this approach some of the performance measures are involved to enhance this work.

\section{A. MEAN SQUARED ERROR (MSE)}

$M S E=\frac{1}{M N} \sum_{i=1}^{M} \sum_{j=1}^{N}(x(i, j)-y(i, j))^{2}$

Where $x(i, j)$ represents the original (reference) image and $y(i, j)$ represents the Segmented image (modified) image and $i$ and $j$ are the pixel position of the $\mathrm{M} \times \mathrm{N}$ image. MSE is zero when $x(i, j)=y(i, j)$.

\section{B. PEAK SIGNAL -TO-NOISE RATIO}

PSNR is the evaluation standard of the reconstructed image quality, and is important feature. The small value of PSNR means that image is poor quality. PSNR is defined as follow

PSNR $=10 \log \frac{255^{2}}{M S E}$

Where 255 is maximum possible value that can be attained by the image signal. Ideally it is infinite. Practically it is in the range of 25 to $40 \mathrm{~dB}$.

\section{NORMALIZED ABSOLUTE ERROR (NAE)}

The large the value of NAE means that image is poor quality.NAE is defined as

$N A E=\frac{\sum_{i=1}^{M} \sum_{j=1}^{M}|x(i, j)-y(i, j)|}{\sum_{i=1}^{M} \sum_{j=1}^{N}|x(i \cdot j)|}$

\section{NORMALIZED CROSS-CORRELATION (NK)}

$N K=\frac{\sum_{i=1}^{M} \sum_{j=1}^{N}(x(i, j) \times y(i, j)}{\sum_{i=1}^{M} \sum_{j=1}^{N}(x(i, j))^{2}}$ 
The performance chart is shown in the fig-3

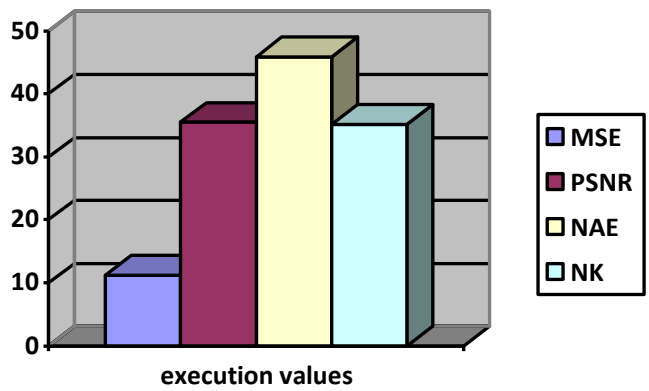

Fig-3 Performance chart

\section{CONCLUSION}

In this paper, detecting a fake currency by using image processing techniques were implemented .This is a sample software to detect the Forgery notes. To convert the images RGB color into HSV value of threshold $=0.3$ and value $=0.4$ and to extract the images to detect the black strips from both image by using the SIFT algorithm the output will be executed finally SVM Classifier classify the fake images.

\section{REFERENCES}

1. Yufeng Kou, Chang-Tien Lu, SiriratSinvongwattana S. ans Yo-Ping Huang,Survey of Fraud Detection Techniques,IEEE International Conference on Networking, Sensing \& Control, 0-78038193-9/04/\$17.0020 2004 IEEE.

2. D. Alekhya, G. DeviSuryaPrabha and G. Venkata Durga Rao, Fake Currency Detection Using Image Processing and Other Standard Methods, International Journal of Research in Computer and Communication Technology, Vol 3, Issue1, January- 2014

3. Rubeena mirza and veenti nanda, Design and Implementation of Indian Paper Currency Authentication System Based on Feature Extraction by Edge Based Segmentation Using Sobel Operator, IJERD, Volume 3, Issue 2 (August 2012), PP. 41-46.

4. Rubeena Mirza and Vinti Nanda, PaperCurrency Verification System Based on Characteristic Extraction Using Image Processing, International Journal of Engineering and Advanced Technology (IJEAT) ISSN: $2249-$ 8958, Volume-1, Issue-3, February 2012.

5. Tanaka M. ; Takeda F. ; Ohkouchi K. and Michiyuki Y. " Recognition Of Paper Currencies By Hybrid Neural Network", IEEE Transactions On Neural Network, Vol: 3, 1998, 1748-1753.

6. Takeda F. and Nishikage T. ," Multiple Kinds Of Paper Currency Recognition Using Neural Network And Application For Euro Currency", IEEE Transactions On Neural Network, Vol.2, 2000, 143-147.

7. Zhang H, Jiang B.,Duan J.H and Bian Z.Z, " Research on Paper Currency Reconition by Neural Networks", Proceedings of the 2nd Int. Conference on Machine Learning and Cybernetics, 2003.

8. Kang J. and Kim H. "New Recognition Algorithm for Various Kinds of Euro Banknotes" Vol: 3, 2003, 2266-2270.

9. A. Vila A., Ferrer N., Mantec'on J. , Bret'on D., Garc'ia J.F., "Development of a fast and nondestructive procedure for characterizing and distinguishing original and fake euro notes", Analytica Chimica Act., Vol: 559,2006, 257-263.

10. Hassanpour, H. ; Yaseri, A. and Ardeshiri, G. , "Feature Extraction For Paper Currency Recognition", IEEE Transactions On Signal Processing And Its Applications, 2007, 1-4.

\section{AUTHORS PROFILE}

M.Praneesh Assistant Professor, Dept of Computer Science, Sri Ramakrishna college of Arts and Science, Bharathiar University.

R.Nagarajan Assistant Professor, Dept of Computer Science, Sri Ramakrishna college of Arts and Science, Bharathiar University.

P.Kavitha Assistant Professor, Dept of Computer Science, Sri Ramakrishna college of Arts and Science, Bharathiar University. 